ARTIGO ORIGINAL

\title{
Estrutura diamétrica e distribuição espacial de Dipteryx odorata (Aubl.) Willd. no oeste do estado do Pará, Brasil
}

\author{
Diametric structure and spatial distribution of Dipteryx odorata (Aubl.) \\ Willd. in western Pará State, Brazil
}

Diego dos Santos Vieira (D), Marcio Leles Romarco de Oliveira ${ }^{1}$ (D) João Ricardo Vasconcellos Gama ${ }^{2}$ (D), Axa Emanuelle Simões Figueiredo ${ }^{3}$ (1) Bruno Oliveira Lafetá ${ }^{4}$ [i]

${ }^{1}$ Universidade Federal dos Vales do Jequitinhonha e Mucuri - UFVJM, Diamantina, MG, Brasil

${ }^{2}$ Universidade Federal do Oeste do Pará - UFOPA, Santarém, PA, Brasil

${ }^{3}$ Instituto Nacional de Pesquisa da Amazônia - INPA, Manaus, AM, Brasil

${ }^{4}$ Instituto Federal de Educação, Ciência e Tecnologia de Minas Gerais - IFMG, São João Evangelista, MG, Brasil

Como citar: Vieira, D. S., Oliveira, M. L. R., Gama, J. R. V., Figueiredo, A. E. S., \& Lafetá, B. O. (2021). Estrutura diamétrica e distribuição espacial de Dipteryx odorata (Aubl.) Willd. no oeste do estado do Pará, Brasil. Scientia Forestalis, 49(131), e3626. https://doi.org/10.18671/scifor.v49n131.11

\begin{abstract}
Resumo
O objetivo deste estudo foi avaliar a estrutura diamétrica e distribuição espacial de Dipteryx odorata (Aubl.) Willd. (cumaru), no estado do Pará, Brasil. O estudo foi realizado em nove Unidades de Trabalho de 100 ha cada, inseridas na Área de Produção Anual nº 8. As áreas estudadas pertencem à Cooperativa Mista da Floresta Nacional do Tapajós, localizada no município de Belterra. Os dados foram coletados por meio de um inventário 100\%, com mapeamento de todos os indivíduos com diâmetro a 1,30 m do solo (DAP) igual ou superior a $20 \mathrm{~cm}$. A estrutura diamétrica foi obtida por meio de análise multivariada. A partir dessa estrutura, testamos cinco funções de densidade probabilísticas (Weibull-3P, Gamma, Normal, Lognormal, Exponencial). O ajuste foi avaliado por meio do teste de Kolmogorov-Smirnov, a 95\% de probabilidade e histogramas. A distribuição espacial foi obtida usando a função K de Ripley. As espécies apresentaram distribuição diamétrica tendendo à normalidade, para indivíduos com DAP $\geq 20 \mathrm{~cm}$. Isso mostra problemas de regeneração, provavelmente causados pela coleta excessiva de sementes de cumaru na década de 1940, baixo número de adultos com alta capacidade regenerativa e baixíssima porcentagem de recrutamento. A distribuição espacial do cumaru é predominantemente aleatória - provavelmente devido ao ciclo de vida da espécie, à medida que os indivíduos crescem, a distribuição torna-se mais aleatória. O boom do cumaru é outra hipótese que apoia a aleatoriedade. A coleta de sementes pode ter afetado o surgimento de novos indivíduos que seriam responsáveis pela agregação esperada para a espécie.
\end{abstract}

Palavras-chave: função K de Ripley, padrão espacial, distribuição diamétrica, cumaru, Amazônia.

\begin{abstract}
The objective of this study was to evaluate the diametric structure and spatial distribution of Dipteryx odorata (Aubl.) Willd. (cumaru), in western Pará State, Brazil. The study was carried out in nine Work Units of 100 ha each, inserted in the Annual Production Area $n^{\circ}$ 8. The studied areas belong to the Tapajós National Forest Mixed Cooperative, municipality of Belterra. We collected the data through a $100 \%$ inventory, with mapping of all individuals with a diameter at $1.30 \mathrm{~m}$ above the ground (DAP) equal to or greater than $20 \mathrm{~cm}$. The diametric structure was obtained through multivariate analysis. From this structure, we tested five probabilistic density functions (Weibull-3P, Gamma, Normal, Lognormal, and Exponential). The adjustment was assessed using the Kolmogorov-Smirnov test, at 95\% probability and
\end{abstract}

Fonte de financiamento: Nenhuma.

Conflito de interesse: Nada a declarar.

Autor correspondente: diegovieir4@gmail.com

Recebido: 11 outubro 2020.

Aceito: 14 dezembro 2020.

Editor: Paulo Henrique Müller Silva.

(c) (i) Este é um artigo publicado em acesso aberto (Open Access) sob a licença Creative Commons Attribution, que permite uso, distribuição e c) reprodução em qualquer meio, sem restrições desde que o trabalho original seja corretamente citado. 
histograms. The spatial distribution was obtained using Ripley's K function. The species had a diametric distribution tending to be normal for individuals with DAP $\geq 20 \mathrm{~cm}$. This suggests problems of regeneration, probably caused by the boom in the collection of cumaru seed in the 1940s; low number of adults with high regenerative capacity and extremely low recruitment percentage. The spatial distribution of cumaru is predominantly random - probably due to the species' life cycle: as the individuals grow, the distribution becomes more random. The cumaru boom is another hypothesis that supports randomness. Seed collection may have affected the emergence of new individuals that would be responsible for the expected aggregation for the species.

Keywords: Ripley's K function, spatial pattern, diametric distribution, cumaru, Amazônia.

\section{INTRODUÇÃO}

A sustentabilidade ecológica do Manejo Florestal Sustentável (MFS) se baseia na premissa de que o uso dos recursos florestais, seja madeireiro ou não madeireiro, mesmo a longo prazo, não deve causar efeitos prejudiciais na reprodução e regeneração das populações manejadas, em comparação com aquelas não manejadas que ocorrem em condições ecológicas semelhantes (Guarino et al., 2014). Além disso, o MFS não deve causar efeitos deletérios na densidade e estrutura populacional de outras espécies na mesma comunidade, bem como na estrutura e nas funções do ecossistema. Mas, para o sucesso do MFS é importante que sejam realizados estudos ecológicos que fornecem bases reais para colheitas sustentáveis, por meio do desenvolvimento de técnicas silviculturais adequadas, ou, até mesmo, para a conservação de espécies (Abreu et al., 2014).

A densidade populacional, estrutura diamétrica e a distribuição espacial estão entre as principais análises realizadas em estudos ecológicos, uma vez que por meio delas é possível fazer inferências sobre o passado, presente e o futuro de espécies arbóreas (Abreu et al., 2014; Lima \& Leão, 2013). A densidade é um fator básico no MFS, pois fornece uma quantificação do volume do recurso madeireiro e não madeireiro em uma determinada área (Herrero-Jáuregui et al., 2012). Isso permite a avalição da viabilidade do MFS, bem como a definição de propósitos econômicos e financeiros (Neves et al., 2016). A estrutura diamétrica também fornece informações sobre o estoque disponível para colheita (Cysneiros et al., 2017), auxilia nas decisões sobre a necessidade de reposição de espécies na comunidade (Abreu et al., 2014) e permite a avaliação da regeneração natural de espécies florestais.

É possível, ainda, por meio da distribuição diamétrica, fazer inferências sobre o ingresso, mortalidade e o histórico de desenvolvimento de espécies (Alves-Junior et al., 2007), determinar sistemas e intensidades de colheita (Orellana et al., 2014), além de criar estratégias de manutenção, recuperação e conservação, de maneira que a comunidade não sofra grandes distúrbios ecológicos (Costa et al., 2019). A distribuição espacial, por sua vez, auxilia no planejamento de atividades de inventário e colheita de madeira, auxiliando na definição do sistema e desenho de amostral utilizado e minimizando custos de locomoção de equipamentos e da frente de trabalho, respectivamente, influenciando diretamente os custos dessas atividades (Moraes et al., 2017; Vieira et al., 2017).

As árvores matrizes, em sistemas de MFS baseados na regeneração natural, também podem ser determinadas por meio do conhecimento da distribuição espacial (Martins et al., 2003). É possível, também, compreender a dinâmica e inferir sobre os processos ecológicos subjacentes e histórico de perturbação de espécies arbóreas (Herrero-Jáuregui et al., 2012). O que se supõem, a partir da densidade populacional, estrutura diamétrica e a distribuição espacial é, dessa forma, fundamental para o bom MFS e conservação de espécies, principalmente aquelas de valor comercial e altos níveis de exploração, como Dipteryx odorata (Aubl.) Willd. (cumaru), que, segundo Pigatti (2017), se destacam dentre as dez espécies com maior número de emissão de guias de colheita, maior volume de madeira e valor de tora comercializada.

A espécie cumaru possui ainda grande valor não madeireiro, pois as amêndoas de seus frutos são aromáticas, de onde se extrai óleo essencial, a cumarina, muito usado na indústria cosmética nacional e internacional (Pinto et al., 2008). A amêndoa é utilizada ainda para curar enfermidades, reduzindo os gastos com medicamentos químicos de custos elevado 
(Rêgo et al., 2017), principalmente para populações tradicionais (Herrero-Jáuregui et al., 2012). O uso múltiplo do cumaru, associado aos altos preços de seus produtos podem acarretar, a longo prazo, a redução de seus populações a baixos níveis, pois nem todos os mecanismos de sustentabilidade são adequadamente abordados por gestores das florestas. O objetivo desse estudo foi avaliar a estrutura diamétrica e o padrão espacial de cumaru com dados de um inventário 100\%, a fim de auxiliar no manejo e conservação dessas espécies na Amazônia.

\section{MATERIAL E MÉTODOS}

\section{Área de estudo}

O estudo foi realizado em nove Unidades de Trabalho (UTs), de 100 ha cada, inseridas na Unidade de Produção Anual (UPA) $n^{\circ} 8$ da Unidade de Manejo Florestal Samambaia ( $3^{\circ} 31^{\prime}$ 01" $\mathrm{S} 55^{\circ}$ 04' 23" O), pertencente à Cooperativa Mista da Floresta Nacional do Tapajós (Coomflona), município de Belterra, oeste do estado do Pará, Brasil. O clima da região, segundo a classificação de Köppen, é do tipo Ami, com temperatura e precipitação média anual de $25,5{ }^{\circ} \mathrm{C}$ e $1.820 \mathrm{~mm}$, respectivamente (Alvares et al., 2013). O relevo é pouco acidentado e apresenta topografia variando de suavemente ondulada a ondulada, com predomínio do Latossolo Amarelo Distrófico (Santos et al., 2017). A vegetação é classificada como Floresta Ombrófila Densa de Terra Firme, caracterizando-se pela dominância de indivíduos arbóreos de grande porte e pela abundância de lianas lenhosas, palmeiras e epífitas.

\section{Coleta de dados}

Os dados foram coletados por meio de um censo florestal, com mapeamento, em coordenadas cartesianas, de todas as espécies com diâmetro a 1,30 m do solo igual ou superior a $20 \mathrm{~cm}$ (DAP $\geq 20 \mathrm{~cm}$ ). O censo incluiu diversas espécies, mas para essa pesquisa foi selecionada apenas a espécie Dipteryx odorata (Aubl.) Willd. (cumaru), devido a sua importância econômica e ecológica na Amazônia. A UPA foi dividida em nove UTs, de 100 ha cada $(1.000 \times 1.000 \mathrm{~m})$, nas quais foram abertas picadas paralelas na direção $\mathrm{N}-\mathrm{S}$, distantes 50 $\mathrm{m}$ entre elas (Vieira et al., 2017). Em cada picada foram instaladas balizas a cada $25 \mathrm{~m}$, com a respectiva metragem em relação à origem, para posterior registro da coordenada $Y$ das árvores (Vieira et al., 2017).

A coordenada $X$ dos indivíduos arbóreos foi obtida por meio da distância entre a árvore e a respectiva linha da picada. Ao final de cada picada, movia-se para a seguinte e executavase a mesma rotina de trabalho, e assim sucessivamente, até completar a última picada da UPA (Vieira et al., 2017). Os indivíduos amostrados foram identificados pelo nome vulgar e coletadas informações sobre o DAP e altura comercial $(\mathrm{Hc})$. O procedimento de identificação das espécies foi inicialmente realizado por mateiros experientes, utilizando-se de observações das folhas, casca, lenho e exsudações. A confirmação da identificação taxonômica foi realizada, posteriormente, por meio de especialistas da Universidade Federal do Oeste do Pará (UFOPA). O sistema de classificação adotado foi o APG IV (Angiosperm Phylogeny Group, 2016).

\section{Análise de dados}

\section{Estrutura diamétrica}

Os dados de DAP foram avaliados por meio das estatísticas básicas (e.g. média, desviopadrão, mediana) e coeficientes de assimetria e curtose, que representam, respectivamente, os graus de afastamento de simetria e achatamento da distribuição de frequência, em relação à distribuição normal. Ambas as medidas são importantes para a descrição da forma e evolução das curvas de distribuição (Cysneiros et al., 2017). A estrutura diamétrica das 
espécies foi obtida por meio de técnicas multivariadas (Vieira et al., 2017). As árvores foram organizadas em ordem crescente de diâmetro e em classes com amplitude de $1 \mathrm{~cm}$, em razão da baixa densidade de indivíduos. Em seguida, elaborou-se uma matriz $\mathrm{D}$ de dados de diâmetro, em que cada variável representava o diâmetro da i-ésima árvore na j-ésima classe de diâmetro. A matriz $D$ foi o input para as análises de agrupamento e discriminante (Vieira et al., 2017).

Os critérios de agrupamento adotados para definir os grupos constituídos por classes de $1 \mathrm{~cm}$ de diâmetro, foram a distância euclidiana e o método de Ward (Souza et al., 2014). A escolha do método multivariado foi pautada na sua maior acurácia para avaliar a distribuição diamétrica de espécies, tendo como objetivo conhecer sua autoecologia, pois as amplitudes das classes diamétricas possuem fundamentos estatísticos válidos. A partir do dendrograma resultante da análise de agrupamento foi traçada uma linha de corte, de modo a formar quinze classes diamétricas. A análise discriminante de Fisher e a estatística Wilk's Lambda foram utilizadas, posteriormente, para confirmação da classificação ou reclassificação dos agrupamentos formados na análise de agrupamento (Souza et al., 2014).

Foram testadas ainda cinco funções de densidade probabilística (FDPs), a saber: Weibull- 3P, Gamma, Normal, Lognormal e Exponencial, para obtenção das distribuições de frequência de árvores em cada classe de diâmetro. Os parâmetros da função Weibull-3P foram obtidos pelo método da máxima verossimilhança, enquanto as demais foram ajustadas pelo método dos momentos. O ajuste foi avaliado por meio do teste de aderência de Kolmogorov-Smirnov, a 95\% de probabilidade. Os dois melhores ajustes foram representados juntos aos histogramas de frequência observada.

\section{Distribuição espacial}

A distribuição espacial foi obtida por meio da função K(s) de Ripley (Ripley, 1981). A função $\mathrm{K}(\mathrm{s})$ de Ripley foi obtida por meio de um círculo com raio $\mathrm{s}$ de $5 \mathrm{~m}$ centrado em cada indivíduo, em que o número de vizinhos presentes na área desse círculo foi contado. Variando o raio $s$ a uma distância máxima de $1.500 \mathrm{~m}$, detectou-se a distribuição espacial da espécie em diferentes escalas de distância. Casos particulares ocorreram quando os indivíduos estavam próximos às bordas da UPA, uma vez que, por ser a função K(s) acumulativa e computar todas as distâncias entre todos os eventos, os indivíduos próximos à borda de raio $s$ maior que o limite do mapa não poderiam ser interpretados como se não houvesse vizinhos. Os vizinhos existiam, mas por estarem fora dos limites da área não foram computados. Consequentemente, o número de indivíduos vizinhos a indivíduos próximos aos limites do mapa seria mais baixo do que para as demais, ocasionando um viés no cálculo do estimador da função K(s) de Ripley.

À vista disso, utilizou-se o estimador da função $\mathrm{K}$ com correção isotrópica de bordadura (1) (Ripley, 1977).

$$
\mathrm{K}(\mathrm{s})=\frac{1}{\hat{\lambda}_{\mathrm{n}}} \sum_{\mathrm{i}=\mathrm{i}=1}^{\mathrm{n}} \sum_{\mathrm{1}}^{\mathrm{n}} \frac{1}{\mathrm{~W}_{\mathrm{I}}}\left(\mathrm{x}_{\mathrm{i}}, \mathrm{x}_{\mathrm{j}}\right) \mathrm{I}\left(\mathrm{x}_{\mathrm{i}}-\mathrm{x}_{\mathrm{j}}<\mathrm{S}\right), \text { para } \mathrm{i} \neq \mathrm{je} \mathrm{S}>0
$$

em que: $n$ é o número de árvores na região de estudo; $X_{i}$ e $X_{j}$ são as coordenadas dos pontos do mapa; $X_{i}-X_{j}$ é a distância euclidiana entre a localização $X_{i}$ e $X_{j}$ i $s$ é um vetor arbitrário de distância; $W_{1} I\left(X_{i}, X_{j}\right)$ é a função de correção para efeito de borda, que representa a proporção da circunferência com centro em $X_{i}$ e com raio $X_{i}-X_{j}$ que está fora da região de estudo; $\hat{\lambda}=\mathrm{n} /|\mathrm{A}|$ é o número de árvores dividido pela área da região de estudos, sendo um estimador não viciado da intensidade do processo; e I(U) é uma função indicadora que assume valor 1 sempre que a condição $U$ for verdadeira e zero quando for falsa.

Objetivando-se analisar os dados graficamente, foram construídos envelopes de confiança por meio de 1.000 simulações Monte Carlo, realizadas com o modelo de completa aleatoriedade espacial (CAE). Posteriormente, foi calculada a função K(s) para os resultados 
das simulações, armazenando-se os valores mínimos e máximos da estimativa de $\mathrm{K}(\mathrm{s})$, utilizados para gerar intervalos de confiança a $99 \%$ de probabilidade. Para facilitar a análise, os valores da função $\mathrm{K}(\mathrm{s})$ foram transformados para $\mathrm{L}(\mathrm{s})$, de acordo com a formulação apresentada a seguir (2), e distribuídos em um gráfico, em que os eixos das abscissas e ordenadas representam, respectivamente, as distâncias $s$ acumuladas e os valores transformados da função K (Ripley, 1977).

$\mathrm{L}(\mathrm{s})=\sqrt{\frac{\mathrm{K}(\mathrm{s})}{\pi}}-\mathrm{s}$

Como resultado, obteve-se envelopes de confiança identificado por duas linhas limites pontilhadas, uma positiva e outra negativa. Se os valores observados de L(s) estiverem dentro dos envelopes construídos, a distribuição espacial é aleatória, caso contrário rejeita-se a hipótese nula e assume-se que a distribuição espacial dos indivíduos é agregada, quando passar do limite superior do envelope, e regular, quando passar do limite inferior (Ripley, 1977). Os cálculos foram realizados com o auxílio do software R versão 3.3.2 (R Core Team, 2018), sendo as análises de agrupamento e discriminante, função K de Ripley e assimetria e curtose obtidas a partir do pacote vegan (Oksanen et al., 2019) splancs (Rowlingson \& Diggle, 2017) e moments (Komsta \& Novomestky, 2015).

\section{RESULTADOS E DISCUSSÃO}

\section{Estrutura diamétrica}

Foram amostrados 0,476 indivíduos ha-1 de cumaru, dos quais 0,380 indivíduos ha-1 apresentaram DAP $\geq 50 \mathrm{~cm}$. O diâmetro variou de 20,7 a 152,8 cm, originando em uma média e coeficiente de variação de $66,3 \mathrm{~cm}$ e $35,2 \%$, respectivamente. A baixa densidade de árvores adultas é a característica mais comum de espécies arbóreas na Amazônia (HerreroJáuregui et al., 2012). O cumaru é considerada uma espécie rara, com 0,476 indivíduos ha-1. A raridade foi determinada com base no conceito de Hubbell \& Foster (1986), que associam raridade à ocorrência de menos de um indivíduo por hectare para uma determinada espécie. A baixa densidade é provavelmente característica da espécie, pois estudos realizados em áreas próximas e distantes da UPA $n^{\circ} 8$ mostraram valores menores do que 1,0 indivíduos ha-1 ${ }^{1}$, até mesmo para níveis de inclusão menores que o adotado na UPA $n^{\circ} 8$, isto é, DAP $\geq 20 \mathrm{~cm}$.

Herrero-Jáuregui et al. (2012), para árvores com DAP $\geq 45 \mathrm{~cm}$, registram 0,15 e 0,11 indivíduos ha-1 de cumaru nas áreas Ambé e Dendrogene, respectivamente, ambas localizadas na Floresta Nacional do Tapajós - FNT, e 0,05 indivíduos ha-1 na área IFT, em Paragominas (PA). Os mesmos autores, considerando outros inventários, porém com DAP $\geq 15 \mathrm{~cm}$, observaram variações de 0,09 a 0,68 indivíduos ha-1 de cumaru para três áreas na FNT. Schulze et al. (2008), em cinco áreas localizadas em diferentes regiões da Amazônia, registram valores que variaram de 0,04 a 0,44 indivíduos ha-1 e 0,03 a 0,37 indivíduos ha-1, para árvores com DAP $\geq 20 \mathrm{~cm}$ e DAP $\geq 50 \mathrm{~cm}$, respectivamente. Cunha et al. (2012) observaram 0,20 indivíduos ha $^{-1}$ de cumaru com DAP $\geq 50 \mathrm{~cm}$, na FNT. Isso mostra que, independentemente do diâmetro de inclusão, a densidade é frequentemente abaixo de 1,0 indivíduos ha-1.

O cumaru possui baixa potencialidade para extração madeireira, pois, considerando-se um diâmetro mínimo de corte de $50 \mathrm{~cm}$ e a manutenção mínima de 3 árvores por 100 hectare com boas características fitossanitárias, houve um saldo de 99 árvores, das quais 58,6\% apresentam uma percentagem de aproveitamento maior do que 50,0\%. O volume registrado para indivíduos com DAP $\geq 50 \mathrm{~cm}$ foi de $1,740 \mathrm{~m}^{3} \mathrm{ha}^{-1}$. Os indivíduos remanescente, podem ser aproveitados para coleta de semente e posterior extração do óleo, rico em cumarina. É importante que as intensidades de colheita madeireira e coleta de sementes não sejam excessivamente altas, pois o cumaru é uma espécie com baixo número de adultos com alta capacidade regenerativa e com taxa de recrutamento extremamente baixa (Herrero- 
Jáuregui et al., 2011). Além disso, deve-se evitar selecionar somente indivíduos de maior diâmetro, pois pode ocorrer a perda da diversidade genética (Jennings et al., 2001) e de grande parte da capacidade de reprodução (Herrero-Jáuregui et al., 2012), e o aumento da distância entre indivíduos adultos, influenciando o processo de polinização e dispersão de sementes na área da UPA $n^{\circ} 8$ e entornos.

O dendrograma, calculado para determinação das classes de diâmetro, mostrou que, considerando uma linha de corte de 3,0\%, foram registrados 15 grupos homogêneos e distintos, denominados de classes diamétricas, as quais apresentaram diferentes amplitudes (Figura 1). A variação de amplitude das classes de DAP é característico desse método, que, segundo Vieira et al. (2017), é preciso e altamente eficiente. Observou-se ainda que o arranjo das árvores individuais em amplitude de 1,0 cm permitiu agrupar, sequencialmente, em ordem crescente as classes diamétricas. A análise discriminante de Fisher entre as classes diamétricas demostraram diferenças significativas para o cumaru $(F=31,212)$, com valores da estatística Wilk's Lambda próximos a zero $\left(\lambda_{\text {wilk }}<0,30\right)$, evidenciando que $98,5 \%$ das classes diamétricas foram classificadas corretamente.


Figura 1. Análise de cluster e estrutura diamétrica observada e estimada pelas funções de densidade probabilística de melhor desempenho para o cumaru, na Floresta Nacional do Tapajós, estado do Pará, Brasil.

A média, mediana e moda do DAP foram de $66,3,63,7$ e $57,3 \mathrm{~cm}$, respectivamente. 0 coeficiente de assimetria obtido para os diâmetros foi de 0,295 , caracterizando a distribuição como assimétrica positiva, inclinada para esquerda, em que o grau de afastamento apresenta valores maiores que a média, que tem valor maior que a mediana, que é maior que a moda. A estrutura diamétrica obtida mostrou uma tendência à normalidade, isto é, baixo número de árvores nas classes diamétricas menores e elevada densidade nas classes intermediárias, com redução acentuada no sentido das maiores classes (Figura 1). Essa distribuição também foi descrita para outras espécies heliófilas, longevas e dominante (Vieira et al., 2017, 2018), geralmente caracterizada por populações flutuantes condicionadas por eventos ocasionais que favorecem a regeneração a longo prazo (Herrero-Jáuregui et al., 2012).

A estrutura diamétrica do cumaru foi descrita por três das cinco FDPs avaliadas, pois os valores de $\mathrm{D}_{\text {CALC }}$ foram maiores que os de $\mathrm{D}_{\text {TAB }}$ para as FDPs Normal, Weibull e Gama, indicando a aceitação da hipótese de nulidade (Tabela 1). A função Normal foi a que demonstrou melhor desempenho, seguida da Weibull-3P e Gamma. As funções Lognormal e Exponencial, por outro lado, apresentaram valores significativos $\left(D_{\text {CALC }}>D_{T A B}\right.$ ), indicando que são inadequadas para obter-se a distribuição diamétrica do cumaru. Isso demonstra também que o cumaru não possui o padrão J-invertido, comumente encontrado em espécies da Amazônia. Cunha et al. (2012), em 800 ha de Floresta Ombrófila Densa, na FNT, registraram uma estrutura diamétrica tendendo à normalidade para indivíduos com DAP $\geq 35 \mathrm{~cm}$. Herrero-Jáuregui et al. (2012), em 
duas outras áreas na FNT, registraram uma estrutura diamétrica com predomínio de indivíduos de tamanho intermediário ou grande. Braz et al. (2012), no estado do Acre, observaram o mesmo comportamento para árvores com DAP $\geq 30 \mathrm{~cm}$.

Tabela 1. Abundâncias e ranking das funções de densidade probabilística ajustadas para o cumaru na Floresta Nacional do Tapajós, estado do Pará, Brasil.

\begin{tabular}{ccccccc}
\hline \multirow{2}{*}{ Espécies } & - & \multicolumn{5}{c}{ Funções de densidade probabilística } \\
\cline { 3 - 7 } & & Gamma & Weibull-3P & Lognormal & Normal & Exponencial \\
\hline \multirow{3}{*}{ D. odorata } & DA & 0,476 & 0,476 & 0,476 & 0,476 & 0,476 \\
& DA $_{\mathrm{EST}}$ & 0,467 & 0,466 & 0,464 & 0,459 & 0,302 \\
& D $_{\mathrm{TAB}}$ & 0,078 & 0,078 & 0,078 & 0,078 & 0,078 \\
& D $_{\text {CAL }}$ & $0,057^{\mathrm{ns}(3)}$ & $0,054^{\text {ns (2) }}$ & $0,097^{*(4)}$ & $0,051^{\mathrm{ns}(1)}$ & $0,208^{*(5)}$ \\
\hline
\end{tabular}

Legenda: $\mathrm{DA}_{\mathrm{EST}}$ e DAOBS = densidade absoluta estimada e observada, respectivamente; $\mathrm{D}_{\mathrm{TAB}}$ e $\mathrm{D}_{\mathrm{CAL}}=\mathrm{D}$ tabelado e calculado, respectivamente, do teste Kolmogorov-Smirnov; ns = não significativo, a 95\% de probabilidade; * =

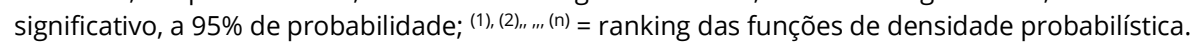

A tendência à normalidade parece ser um comportamento característico do cumaru, o que indica um provável desequilíbrio entre mortalidade e recrutamento, causados por possíveis distúrbios antropogênicos passados ou naturais. Se a mesma área fosse inventariada considerando um DAP $\geq 10 \mathrm{~cm}$, provavelmente, o desequilíbrio continuaria ocorrendo, uma vez que as novas árvores inventariadas não compensariam o baixo número de árvores por hectare observado nas classes diamétricas iniciais. Isso porque em áreas próximas à UPA $n^{\circ} 8$, Herrero-Jáuregui et al. (2012) encontraram poucas $(0,09$ árv. ha-1) ou nenhuma árvores $(0,0$ árv. ha-1) com DAP de 15 a $30 \mathrm{~cm}$, e muito poucas na classe diamétrica de $30-45 \mathrm{~cm}$. Os mesmos autores afirmam ainda que esse comportamento, aliado à maior densidade de árvores nas classes de diâmetro intermediárias, pode ser associado ao boom na coleta de semente de cumaru, na década de 1940, e ao baixo número de adultos com alta capacidade regenerativa.

A baixa ocorrência de indivíduos de cumaru nas classes de $20-36 \mathrm{~cm}$ e $36-44 \mathrm{~cm}$ é preocupante, devido à baixa capacidade regenerativa e baixo número de mudas (HerreroJáuregui et al., 2011), o que reduz de maneira drástica a percentagem de recrutamento. Herrero-Jáuregui (2009), em transcetos de 1,0 ha, encontraram uma média de seis recrutas por hectare. 0 mesmo autor registrou que apenas $1 / 3$ das árvores de cumaru contribuem para produção de mudas. Isso têm consequências negativas para o manejo de cumaru, porque percentuais de coleta de sementes inadequadas, seleção de árvores matrizes com baixo potencial reprodutivo e altas intensidade de colheita de madeira mudariam a dinâmica da população, pois a fonte mais eficiente de novos indivíduos seria eliminada da população. É preciso, dessa forma, atenção durante o MFS de cumaru.

\section{Padrão espacial}

A função K de Ripley demonstrou que a espécie cumaru atende parcialmente à hipótese de completa aleatoriedade espacial, pois a distribuição espacial variou em função da distância máxima de $1.500 \mathrm{~m}$. Até distâncias de $\pm 60 \mathrm{~m}$ a distribuição foi aleatória, passando para uma leve agregação até $\pm 465 \mathrm{~m}$, e a partir daí a distribuição dos indivíduos na UPA $n^{\circ} 8$ foi completamente aleatória (Figura 2). A distribuição espacial predominante, nesse caso, foi a aleatória. A aleatoriedade do cumaru foi semelhante à registrada por Herrero-jáuregui et al. (2012), avaliando indivíduos com DAP $\geq 10 \mathrm{~cm}$ e DAP $\geq 45 \mathrm{~cm}$, na Floresta Nacional do Tapajós (PA). Souza et al. (2011), também registraram distribuição aleatória na Floresta Estadual do Amapá (AP), mas Barroso et al. (2007) observaram agregação, ambos considerando DAP $\geq 10 \mathrm{~cm}$. 

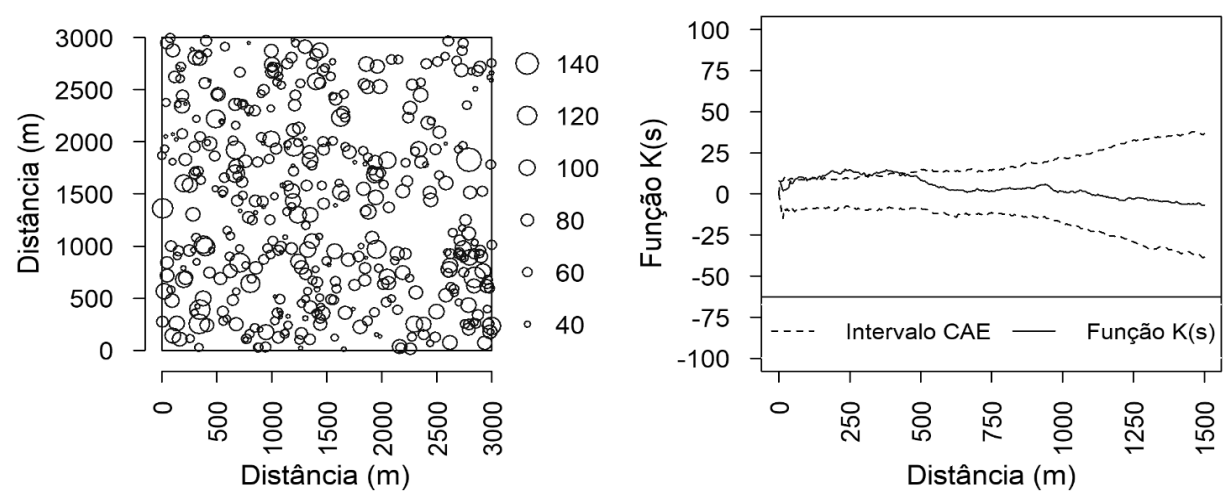

Figura 2. Processo pontual marcado (DAP) e função K de Ripley para indivíduos de cumaru na Floresta Nacional do Tapajós, estado do Pará, Brasil.

A aleatoriedade não era esperada, uma vez que a distribuição espacial é fortemente determinada pelas síndromes de dispersão (Negrini et al., 2012; Nyiramana et al., 2011; Seidler \& Plotkin, 2006; Vieira et al., 2017, 2018). O cumaru, possui dispersão barocórica e zoocórica. Logo, se esperava uma distribuição agregada. Isso devido, normalmente, essas síndromes limitarem a distribuição dos propágulos, causando altas taxas de germinação e recrutamento em áreas próximas às árvores mãe, enquanto em outras áreas esses processos são menores e aleatórios (Negrini et al., 2012; Vieira et al., 2018). A leve agregação foi registrada somente para distâncias de 60 - $465 \mathrm{~m}$, indicando pequenos agrupamentos são formados por três a quatro árvores, em sua maioria com diâmetros de $40-90 \mathrm{~cm}$, conforme se pode observar no processo de pontos marcados do cumaru (Figura 1).

As variações na distribuição espacial do cumaru, em função da distância, podem ser relacionadas ao porte arbóreo dos indivíduos, uma vez que os mais jovens tendem a ocorrer mais próximos uns dos outros, formando agregados (Araújo et al., 2014). À medida que crescem, a alta taxa de mortalidade dependente da densidade e o aumento da competição interespecífica, faz com que pouco indivíduos de cumaru atinjam os maiores diâmetros, ficando mais distanciados uns dos outros, o que leva à aleatoriedade. Isso mostra que, provavelmente, o padrão de cumaru muda ao longo de seu ciclo de vida. HerreroJáuregui et al. (2012) sugerem que essa mudança de agregado para aleatório ocorra na fase de plântulas, pois, ao avaliar indivíduos com DAP $\geq 10 \mathrm{~cm}$, o padrão já era aleatório. Isso explica, em partes, a distribuição aleatória registrada para cumaru na UPA n 8.

Outra hipótese é que a distribuição aleatória seja decorrência o boom de cumaru na década de 1940 (Amorim, 2000), pois, provavelmente, a coleta de sementes nessa época foi tão intensa que não permitiu o recrutamento de novos indivíduos. Isso explicaria a alta ocorrência de indivíduos com diâmetro entre 40 e $90 \mathrm{~cm}$, que provavelmente encontram-se estabelecidos na época. É recomendado, nesse caso, que os níveis de coleta de sementes em populações de cumaru sejam médios, e que sejam aplicados tratamentos silviculturais, como o enriquecimento, para que a viabilidade natural dessa população não seja comprometida. Herrero-Jáuregui et al. (2012) não registraram indivíduos de D. odorata nas menores classes diamétricas, em uma área de coleta de sementes na Floresta Nacional do Tapajós, o que reforça a suposição de que a ausência de indivíduos menores seja produto da coleta intensiva de semente de cumaru.

A distribuição aleatória de indivíduos de cumaru dificulta o acesso aos produtos madeireiros e não madeireiros. As trilhas para coletas de sementes tornam-se maiores, demoradas e mais difíceis de serem programadas. Os ramais de arraste de madeira tornamse mais densos, aumentando os impactos sobre a vegetação remanescente. A aplicação de tratamentos silviculturais, como o raleamento ou enriquecimento, para o favorecimento de indivíduos estabelecidos e aumento na densidade de plântulas, se tornam mais difíceis do que se a distribuição fosse agregada. A agregação tornaria o processo de colheita menos oneroso e mais sistêmico (Costa et al., 2019). O MFS do cumaru, diante das características 
estruturais e espaciais expostas, se torna mais dificultoso, o que, provavelmente, afetaria a reposição de indivíduos e do volume para ciclos de corte de 30 anos. Dias (2001), sugere que espécies com essas características sejam manejadas a nível de indivíduo não de população.

\section{CONCLUSÃO}

A espécie cumaru apresentou distribuição diamétrica tendendo à normalidade para indivíduos com DAP $\geq 20 \mathrm{~cm}$, o que sugere problemas de regeneração ocasionados, provavelmente, pelo excesso na coleta de semente de cumaru, na década de 1940, baixo número de adultos com alta capacidade regenerativa e percentagem de recrutamento extremamente baixa.

A distribuição espacial do cumaru é predominantemente aleatória. É provável que a aleatoriedade seja decorrência do ciclo de vida da espécie, pois a medida que os indivíduos crescem sua distribuição torna-se mais aleatória. O boom do cumaru, na década de 1940, é outra hipótese que sustenta a aleatoriedade, pois a coleta de sementes teria afetado o surgimento de novos indivíduos que seriam os responsáveis pela agregação esperada para o cumaru.

É importante a realização de estudos que determinem taxas de coleta de sementes que não afetem a regeneração dessa espécie, além da avaliação e determinação de tratamentos silviculturais que favoreçam seu desenvolvimento, assegurando sua presença na estrutura da floresta a longo prazo. Avaliações em níveis regionais mostrariam se os problemas são locais ou comuns da espécies. Isso mostraria a importância de instruções normativas para o manejo florestal não madeireiro do cumaru.

\section{REFERÊNCIAS BIBLIOGRÁFICAS}

Abreu, J. C., Guedes, M. C., Guedes, A. C. L., \& Batista, E. M. (2014). Estrutura e distribuição espacial de andirobeiras (Carapa spp.) em floresta de várzea do estuário amazônico. Ciência Florestal, 24(4), 1009-1019. http://dx.doi.org/10.5902/1980509816614.

Alvares, C. A., Stape, J. L., Sentelhas, P. C., de Moraes Gonçalves, J. L., \& Sparovek, G.. (2013). Köppen's climate classification map for Brazil. Meteorologische Zeitschrift (Berlin), 22(6), 711-728. http://dx.doi.org/10.1127/0941-2948/2013/0507.

Alves-Junior, F. T., Lins, C. F., Brandão, S., Rocha, K. D., Silva, J. T., Marangon, L. C., \& Ferreira, R. L. C. (2007). Estrutura diamétrica e hipsométrica do componente arbóreo de um fragmento de Mata Atlântica, Recife-PE. Cerne, 13(1), 83-95.

Amorim, A. T. S. (2000). Santarém: uma síntese histórica. (254p.). Santarém: Editora ULBRA.

Angiosperm Phylogeny Group. (2016). An update of the Angiosperm Phylogeny Group classification for the orders and families of flowering plants: APG IV. Botanical Journal of the Linnean Society, 181(1), 1 20. http://dx.doi.org/10.1111/boj.12385.

Araújo, E. J. G., David, H. C., Péllico Neto, S., Morais, V. A., \& Scolforo, J. R. S. (2014). Padrão espacial de espécies arbóreas em fragmento de floresta estacional semidecidual. Revista de Ciências Agrárias (Belém), 57(2), 166-171. http://dx.doi.org/10.4322/rca.2014.010.

Barroso, J. G., Lima, D. V. M., Silveira, M., \& Salimon, C. (2007). Estrutura populacional e distribuição espacial de Aspidosperma vargasii (Apocynaceae) e Dipteryx odorata (Fabaceae) em uma floresta ombrofila aberta com bambu no estado do Acre. In Anais do $8^{\circ}$ Congresso de Ecologia do Brasil. Caxambu: Sociedade de Ecologia do Brasil.

Braz, E. M., Thaines, F., Mattos, P. P., Ruy, C. C., Canetti, A. \& Zachow, R. (2012). Considerações sobre o manejo da estrutura diamétrica do cumarú-ferro (Dipteryx odorata), em empresa no estado do Acre (Comunicado Técnico, Vol. 298, pp. 1-5). Colombo: Embrapa Florestas.

Costa, S. N., Rabelo, F. G., Lima, R. B., Silva, D. A. S., Lima, C. W. P., \& Santos, S. M. (2019). Estrutura populacional de Manilkara huberi (Ducke) A. Chev. e Dinizia excelsa Ducke em floresta de terra firme no Amapá. Nativa (Sinop), 7(4), 445-451. http://dx.doi.org/10.31413/nativa.v7i4.7638.

Cunha, U. S., Machado, S. A., Figueiredo-Filho, A., \& De Freitas, J. V. (2012). Diameter structure and phytosociological frequency of a "terra firme" amazon forest, before and after selective cutting. Floresta, 42(2), 293-304. http://dx.doi.org/10.5380/rf.v42i2.21463. 
Cysneiros, V. C., Amorim, T. D. A., Mendonça Júnior, J. D. O., Gaui, T. D., De Moraes, J. C. R., Braz, D. M., \& Machado, S. D. A. (2017). Distribuição diamétrica de espécies da Floresta Ombrófila Densa no Sul do Estado do Rio de Janeiro. Pesquisa Florestal Brasileira, 37(89), 1-10. http://dx.doi.org/10.4336/2017.pfb.37.89.1070.

Dias, A. S. (2001). Consideraciones sociales y silviculturales para el manejo forestal diversificado en una comunidad riberiña en la "Floresta Nacional do Tapajós, Amazônia brasileña (Magíster Scientiae). Centro Agronómico Tropical de Investigación y Enseñanza, Turrialba, Costa Rica.

Guarino, E. D. S. G., Gessner, C. M., Wadt, L. H. O., Fonseca, F. L., \& Raposo, A. (2014). Estrutura etária e espacial de uma população natural de Carapa guianensis Aubl.(Meliaceae) na Amazônia Sul Ocidental. Scientia Forestalis, 42(101), 91-99.

Herrero-Jáuregui, C. (2009). Gestión integrada de los recursos forestales en el bosque tropical húmedo: ecología de dos especies de uso múltiple (Doctor Scientiae). Universidad Complutense de Madrid, Madrid.

Herrero-Jáuregui, C., García-Fernández, C., Sist, P. L. J. \& Casado, M. A. (2011). Recruitment dynamics of two low-density neotropical multiple-use tree species. Plant Ecology, 212, 1501. http://dx.doi.org/10.1007/s11258-011-9924-0.

Herrero-Jáuregui, C., Sist, P., \& Casado, M. A. (2012). Population structure of two low-density neotropical tree species under different management systems. Forest Ecology and Management, 280, 31-39. http://dx.doi.org/10.1016/j.foreco.2012.06.006.

Hubbell, S. P., \& Foster, R. B. (1986). Commonness and rarity in a neotropical forest: implications for tropical tree conservation. In M. Soule (Ed.), Conservation biology: the science of scarcity and diversity (pp. 205-231). Saunderland: Sinauer Associates.

Jennings, S. B., Brown, N. D., Boshier, D. H., Whitmore, T. C. \& Lopes, J. C. A. (2001). Ecology provides a pragmatic solution to the maintenance of genetic diversity in sustainably managed tropical rain forests. Forest Ecology and Management, 154(1-2), 1-10. http://dx.doi.org/10.1016/S03781127(00)00637-X.

Komsta, L. \& Novomestky, F. (2015). moments: Moments, cumulants, skewness, kurtosis and related tests. Versão 0.14. Vienna, Austria: R Foundation for Statistical Computing.

Lima, J. P. C., \& Leão, J. R. A. (2013). Dinâmica de crescimento e distribuição diamétrica de fragmentos de florestas nativa e plantada na Amazônia Sul Ocidental. Floresta e Ambiente, 20(1), 70-79. http://dx.doi.org/10.4322/floram.2012.065.

Martins, S. S., Couto, L., Machado, C. C., \& Souza, A. L. (2003). Efeito da exploração floresta seletiva em uma floresta estacional semidecidual. Revista Árvore, 27(1), 65-70. http://dx.doi.org/10.1590/S010067622003000100009.

Moraes, G. C., Schorr, L. P. B., Aguiar, J. T., Cuchi, T., \& Melo, L. O. (2017). Mudanças na estrutura diamétrica e no arranjo espacial de Manilkara elata em área manejada na Floresta Nacional do Tapajós. Enciclopédia Biosfera, 14(25), 950-959. http://dx.doi.org/10.18677/EnciBio_2017A76.

Negrini, M., Aguiar, M. D., Vieira, C. T., Silva, A. C., \& Higuchi, P. (2012). Dispersão, distribuição espacial e estratificação vertical da comunidade arbórea em um fragmento florestal no Planalto Catarinense. Revista Árvore, 36(5), 919-930. http://dx.doi.org/10.1590/S0100-67622012000500014.

Neves, E. S., Wadt, L. H. O., \& Guedes, M. C. (2016). Estrutura populacional e potencial para o manejo de Bertholletia excelsa (Bonpl.) em castanhais nativos do Acre e Amapá. Scientia Forestalis, 44(109), http://dx.doi.org/10.18671/scifor.v44n109.02.

Nyiramana, A., Mendoza, I., Kaplin, B. A., \& Forget, P. M. (2011). Evidence for seed dispersal by rodents in tropical montane forest in Africa. Biotropica, 43(6), 6, 654-657. http://dx.doi.org/10.1111/j.17447429.2011.00810.x.

Oksanen, J., Blanchet, F. G., Friendly, M., Kindt, R., Legendre, P., McGlinn, D., Minchin, P. R., O'Hara, R. B., Simpson, G. L., Solymos, P., Henry, M., Stevens, H., Szoecs, E., \& Wagner, H. (2019). vegan: Community Ecology Package. Versão 2.5-6. Vienna, Austria: R Foundation for Statistical Computing.

Orellana, E., Figueiredo Filho, A., Péllico-Netto, S., \& Dias, A. N. (2014). Modelagem da distribuição diamétrica de espécies florestais em um fragmento de floresta ombrófila mista. Revista Árvore, 38(2), 297-308. http://dx.doi.org/10.1590/S0100-67622014000200010.

Pigatti, L. O. (2017). Diagnóstico do setor florestal no período de 2007 a 2015 no estado do Pará (Bacharelado). Universidade Federal Rural da Amazônia, Belém.

Pinto, A. M., Morellato, L. P. C., \& Barbosa, A. P. (2008). Fenologia reprodutiva de Dipteryx odorata (Aubl.) Willd (Fabaceae) em duas áreas de floresta na Amazônia Central. Acta Amazonica, 38(4), 643649. http://dx.doi.org/10.1590/S0044-59672008000400006. 
R Core Team. (2018). R: a language and environment for statistical computing. Versão 3.5.1. Vienna, Austria: R Foundation for Statistical Computing.

Rêgo, L. J. S., Silva, M. L., Silva, L. F., Gama, J. R. V., Reis, L. P., \& Reis Reis, P. C. (2017). Caracterização do consumo de amêndoa de cumaru na Amazônia Oriental. Biota Amazônia, 7(3), 23-27. http://dx.doi.org/10.18561/2179-5746/biotaamazonia.v7n3p23-27.

Ripley, B. D. (1977). Modelling spatial patterns. Journal of the Royal Statistical Society. Series B. Methodological, 39(2), 172-192. http://dx.doi.org/10.1111/j.2517-6161.1977.tb01615.x.

Ripley, B. D. (1981). Spatial statistics (252 p.). London: John Wiley \& Sons.

Rowlingson, B. \& Diggle, P. (2017). Splancs: Spatial and Space-Time Point Pattern Analysis. Versão 2.01-40. Vienna, Austria: R Foundation for Statistical Computing.

Santos, L. S., Martorano, L. G., Gutierrez, C. B. B., Pontes, A. N., Silva, O. M. \& Gutierrez, D. M. G. (2017). Aspecto fisiográficos da Floresta Nacional do Tapajós e seu entorno - oeste do Pará, Brasil. Revista Espacios, 38(2), 26.

Schulze, M., Grogan, J., Landis, R. M., \& Vidal, E. (2008). How rare is too rare to harvest?: management challenges posed by timber species occurring at low densities in the Brazilian Amazon. Forest Ecology and Management, 256(7), 1443-1457. http://dx.doi.org/10.1016/j.foreco.2008.02.051.

Seidler, T. G., \& Plotkin, J. B. (2006). Seed dispersal and spatial pattern in tropical trees. PLoS Biology, 4(11), 2132-2137. PMid:17048988. http://dx.doi.org/10.1371/journal.pbio.0040344.

Souza, A. L., Medeiros, R. M., Matos, L. M. S., Silva, K. R., Corrêa, P. A., \& Faria, F. N. (2014). Estratificação volumétrica por classes de estoque em uma floresta ombrófila densa, no município de Almeirim, Estado do Pará, Brasil. Revista Árvore, 38(3), 533-541. http://dx.doi.org/10.1590/S010067622014000300016.

Souza, R. N., Aparício, P. S., Aparício, W. C. S., Doff, S. E., Guedes, M. C., \& Oliveira, L. P. S. (2011). Distribuição diamétrica, espacial, e volumetria de Dipteryx odorata (Aubl.) Willd (Cumarú) na Florestal Estadual do Amapá - FLOTA - AP. In Anais do $5^{\circ}$ Simpósio Latino-Americano sobre Manejo Florestal (pp. 714-720). Santa Maria: UFSM.

Vieira, D. S., Oliveira, M. L. R., Vasconcelos Gama, J. R. V., Machado, E. L. M., \& Görgens, E. B. (2017). Patrón espacial y métodos de muestreo para Bertholletia excelsa (castaña de Pará) en la Mesorregión del Bajo Amazonas, estado de Pará, Brasil. Bosque (Valdivia), 38(1), 97-107. http://dx.doi.org/10.4067/S0717-92002017000100011.

Vieira, D. S., Oliveira, M. L. R., Gama, J. R. V., Oliveira, B. L., Rego, A. K. C., \& Bezerra, T. G. (2018). Processos de amostragem para Carapa guianensis Aubl. na Amazônia. Cerne, 24(3), 169-179. http://dx.doi.org/10.1590/01047760201824032514.

Contribuição dos Autores: DSV: conceituação, curadoria de dados, análise formal, metodologia, supervisão, escrita - primeira redação, escrita revisão e edição; MLRO, JRVG e BOL: supervisão, escrita - primeira redação, escrita - revisão e edição; e AESF: supervisão e escrita - primeira redação. 